\title{
COMPOSIÇÃO FLORÍSTICA E PARÂMETROS ESTRUTURAIS DE UM HECTARE DE FLORESTA DENSA DE TERRA FIRME NO RIO UATUMÃ, AMAZÔNIA, BRASIL
}

\author{
Iêda Leão do AMARAL*, F ${ }^{\text {ca. }}$ Dionizia A. MATOS*, José LIMA*
}

\begin{abstract}
RESUMO - Foi inventariada uma área de floresta densa de terra firme no rio Uatumã, município de São Sebastião, Estado do Amazonas (2 20' $04^{\prime \prime}$ S e $58^{\circ} 45^{\prime} 26^{\prime \prime}$ W), objetivando estudar a composição floristica, riqueza de espécies e parâmetros estruturais da vegetação. Executou-se o levantamento de um hectare, empregando-se um transecto de $10 \times 1000 \mathrm{~m}$, dividido em 20 parcelas de $10 \times 50 \mathrm{~m}$, onde mensuraram-se todos os indivíduos com DAP $\geq 10 \mathrm{~cm}$, incluindo árvores, cipós e palmeiras. $\mathrm{O}$ estrato inferior foi abordado em pequenas parcelas de $2 \times 2 \mathrm{~m}$, obedecendo o critério de categoria de tamanho. Os parâmetros estruturais foram avaliados por meio do IVI (Índice de Valor de Importância) das espécies, número de indivíduos por classe de diâmetro e espectro biológico do estrato inferior. Foram registrados 741 individuos (com DAP $\geq 10 \mathrm{~cm}$ ), distribuidos em 46 famílias, 118 gêneros e 145 espécies. As familias com maior número de espécies foram Leguminosae sensu lato (33), Myrtaceae (8) e Lauraceae (7), representando 33\% do total de indivíduos registrados. Protium apiculatum e Eschweilera coriacea foram as espécies com maior IVI (22,4 e 17,6 respectivamente), representando 15\% do total. Estas duas espécies foram as que apresentaram maior uniformidade de distribuição e maior número de individuos na área. O estrato inferior é composto de alta proporção de individuos $(28,7 \%)$, cujas espécies representam $25,4 \%$ do total de espécies registradas que têm seu ciclo de vida neste estrato. Portanto, estas espécies não podem ser incluídas como parte da regeneração florestal, como é considerada por muitos projetos de manejo florestal.
\end{abstract}

Palavras-chave: Florística, Estrutura, Riqueza de espécies, Amazonas, Rio Uatumã

\section{Structural Parameters and Floristic Composition of One Hectare of Terra Firme Dense Forest in the Uatumã River Valley, Amazonia, Brazil}

ABSTRACT - An area of terra firme dense forest in the Uatumã River basin, county of São Sebastião, Amazonas, Brazil ( $2^{\circ} 20^{\prime} 04^{\prime \prime} \mathrm{S}$ and $\left.58^{\circ} 45^{\prime} 26^{\prime \prime} \mathrm{W}\right)$ was surveyed. The floristic composition, richness of species, and structural parameters of the vegetation were studied. A survey of one hectare was carried out by using a transect of $10 \times 1000 \mathrm{~m}$, divided in 20 plots of $10 \times 50$ $\mathrm{m}$, where every individual with a diameter at breast height $(\mathrm{DBH}) \geq 10 \mathrm{~cm}$ was measured, including trees, lianas and palms. The understory stratum was studied by using small plots of $2 x$ $2 \mathrm{~m}$, according to the size category criterion. Structural parameters were assessed through the Importance Value Index (IVI) of the species, the number of individuals per class of diameter, and the biological spectrum of the understory stratum. A total of 741 individuals (with DBH $\geq$ $10 \mathrm{~cm}$ ) were recorded, distributed in 46 families, 118 genera and 145 species. Families with the greatest number of species were Leguminosae sensu lato (33), Myrtaceae (8) and Lauraceae (7), representing $33 \%$ of the total recorded individuals. Protium apiculatum and Eschweilera coriacea were the species with higher IVI (22.4 and 17.6 respectively), representing $15 \%$ of the total IVI. These two species presented more uniform distributions and greatest number of individuals in the area. The understory stratum was composed of a high proportion $(28.7 \%)$ of individuals, corresponding to $25.4 \%$ of total species, which have their whole life circle in this stratum. Therefore, these species should not be included as part of forest regeneration, as practiced by many projects of forest management.

Key-words: Floristics, Structure, Species richness, Amazonia, Uatumã River

* Instituto Nacional de Pesquisas da Amazônia (INPA), Coordenação de Pesquisas em Botânica (CPBO), Av. André Araújo, 2936, Petrópolis, CEP 69.083-000, Manaus, AM. Brasil, e-mail: iamaral@inpa.gov.br 


\section{INTRODUÇÃO}

O Rio Uatumã, de águas escuras, é afluente da margem esquerda do Rio Amazonas. É estreito na jusante e mais largo na montante, constituindo um verdadeiro reservatório de compensação para o rio Amazonas, que para ele reflui suas águas quando está cheio e dele as recebe quando seu nivel baixa (Albuquerque, 1922). Suas margens em alguns trechos são altas e compostas de argila terciária; a uma certa altura começam a aparecer praias e seixos z Inundável (Caatinga-gapó) segundo a classificação brasileira, adaptada a um sistema universal (Veloso et al., 1991).

Este rio está atualmente numa situação crítica, pois, com o desenvolvimento econômico da região Norte, diversas atividades altamente impactantes localizam-se ao longo de suas margens. Por exemplo, na década de 70, a mineradora Paranapanema iniciou extração de cassiterita e construiu uma hidroelétrica em um dos seus principais afluentes, o rio Pitinga. Na década de 80 , a Eletronorte construiu a hidroelétrica de Balbina no meio do seu percurso, formando um grande lago raso e uma barreira hidrográfica. Atualmente, a extração de seixos ocorre tanto nas margens quanto no leito para utilização na construção civil de Manaus.

Para subsidiar a conservação, o conhecimento dos ecossistemas do Rio Uatumã é de fundamental importância. Por isto, elaborou-se o presente estudo, objetivando conhecer a composição florística, a riqueza de espécies e os parâmetros estruturais da vegetação predominante na área mencionada.

\section{MATERIAL E MÉTODOS}

\section{Área de estudo}

O presente estudo foi desenvolvido em floresta densa de terra firme, de solos argilosos, localizada na bacia do Rio Uatumã, município de São Sebastião do Uatumã, Estado do Amazonas, situada ao redor das coordenadas $02^{\circ} 20^{\prime} 04^{\prime \prime}$ $\mathrm{S}$ e $58^{\circ} 45^{\prime} 26^{\prime \prime} \mathrm{W}$ (Fig. 1).

\section{Métodos}

\section{a) campo}

Foi inventariado um hectare em um transecto de $10 \times 1000 \mathrm{~m}$, dividido em 20 parcelas de $10 \times 50 \mathrm{~m}$, onde mensurou-se todos os indivíduos arbóreos, palmeiras e lianas lenhosas (cipós) com DAP (Diâmetro à Altura do Peito) $\geq 10 \mathrm{~cm}$. A abordagem do estrato inferior (sub-bosque) foi em pequenas parcelas de $2 \times 2 \mathrm{~m}$, alocadas no final de cada parcela de 10 x $50 \mathrm{~m}$, obedecendo ao critério de categoria de tamanho usado por Higuchi et al. (1985), Em seguida, coletou-se material botânico de todos os espécimes abordados, tanto do estrato superior, quanto do inferior, para identificação das espécies.

A aplicabilidade medicinal e madeireira das espécies foram obtidas a partir de informações prestadas por moradores locais, bem como em Silva et al. (1977), e quando esta não era conhecida, classificou-se como de potencial ecológico. 


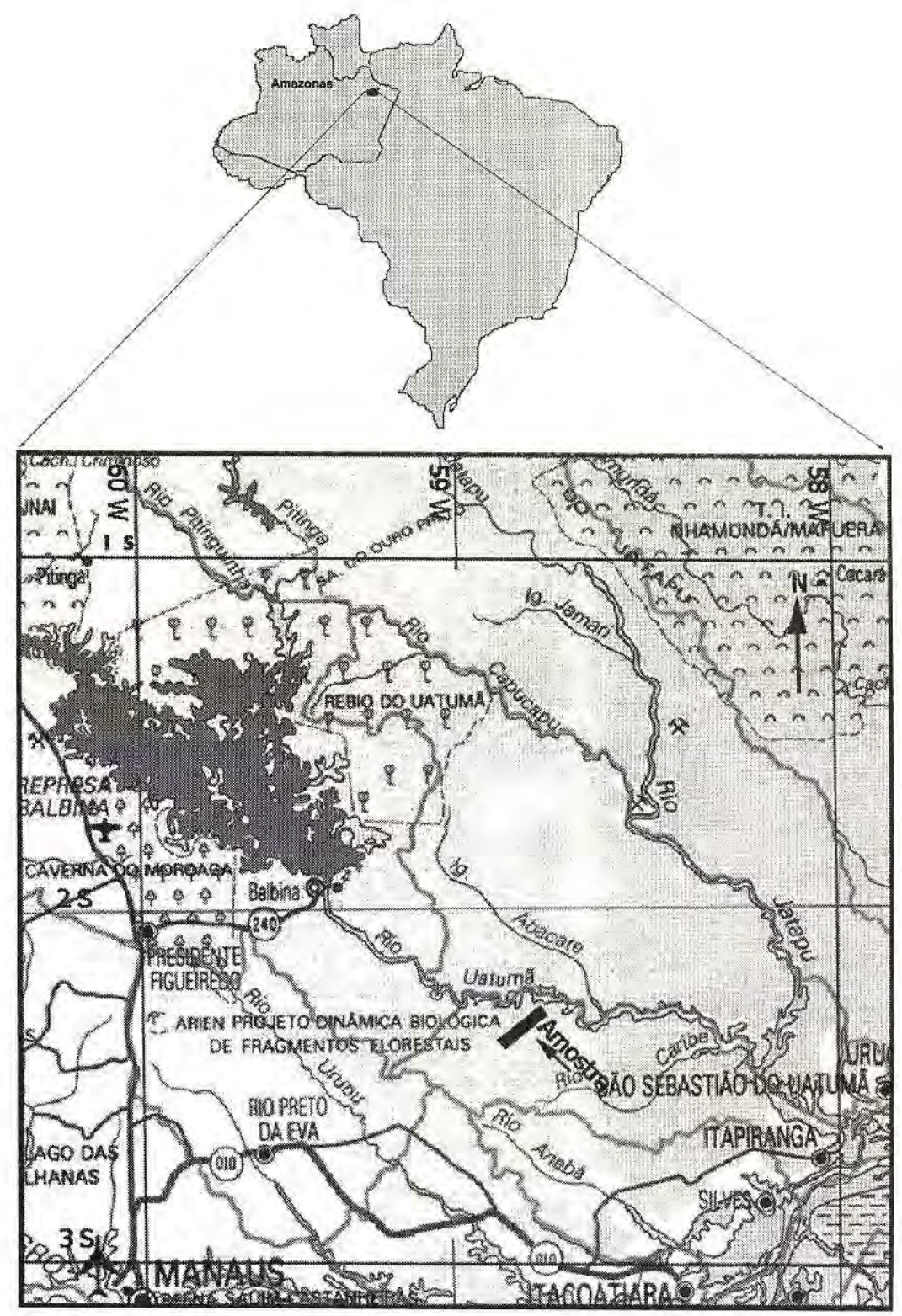

Figura 1. Localizaçăo da área de estudo no Rio Uatumă, AM, Brasil. 


\section{b) laboratório}

O material botânico coletado passou por um processo de desidratação, após o qual cada amostra foi identificada por comparação com as amostras existentes no acervo do herbário do INPA. Após as identificações, foram feitas as análises de composição florística, por meio do número de famílias, gêneros, espécies e riqueza de espécies, tomando por base a curva espécie/área que, segundo Magurran (1988) e Condit et al. (1996), é eficiente para avaliar a riqueza de uma comunidade florística. Parâmetros estruturais foram avaliados por meio do IVI (Índice de Valor de Importância) e número de indivíduos por classe de DAP. A análise do estrato inferior foi efetuada pela classificação de categoria de tamanho, adotada por Higuchi et al. (1985), e das formas de vida (hábito), adaptada de Aubréville (1963). O material botânico fértil coletado foi depositado no acervo do herbário do INPA; os estéreis serão preservados por cinco anos após a publicação dos resultados.

\section{RESULTADOS E DISCUSSÃO}

Aspecto fisionômico da floresta ombrófila densa de terra firme

Esta formação vegetal possui uma fisionomia exuberante, com grande número de árvores altas e finas, e um reduzido número de espécimes grossos. A classe de DAP dominante é de $15 \mathrm{~cm}$, com 330 indivíduos, representando em torno de $44 \%$ do total registrado.
O sub-bosque em geral é denso, com concentração de pequenas palmeiras como espinho-preto (Astrocaryum sociale) e mumbaca (A. gynacanthus). As espécies lenhosas mais freqüentes são branquinha (Rinorea racemosa), erva-de-rato (Psychotria sp.), orelha-de-burro (Miconia sp.), Solanum sp., capitiú (Siparuna sp.), algumas herbáceas, como arumã (Ischnosiphon ovatus), gramíneas (Pariana sp.) e pteridófitas. Nas clareiras naturais houve um adensamento de cipós, sendo os mais comuns escada-de-jaboti (Bauhinia macrostachya) e timbó (Derris floribunda).

O estrato médio, representado por indivíduos com altura de 3 a $9 \mathrm{~m}$, onde ocorre a classe dominante de DAP $(15 \mathrm{~cm})$, apresenta-se denso, dominado por espécies como sucuuba (Himatanthus sucuuba), Payparola sp., ubaia (Calyptranthes bipennis), cacauí (Theobroma speciosum) e tinteiro (Miconia regelii). Neste estrato, observou-se maior concentração de epifitas, principalmente orquidáceas, aráceas, bromeliáceas, gesneriáceas e pteridófitas.

O dossel, estrato superior, possui aspecto rugoso, devido às árvores mais altas e o dissecamento do relevo. As árvores apresentam copas globosas, próximas umas das outras, que dificulta a penetração de luz; as emergentes são escassas, não ultrapassando a $35 \mathrm{~m}$ de altura, tendo como representantes cupiúba (Goupia 
glabra), angelim-rajado (Zygia racemosum), matá-matá (Eschweilera coriacea), castanha-do-brasil (Bertholletia excelsa), pajurá-da-mata (Parinari montana), taxi-preto (Tachigali myrmecophila) e piquiá (Caryocar villosum). As palmeiras mais freqüentes foram bacaba (Oenocarpus bacaba) e inajá (Maximiliana maripa). Quando do levantamento, observou-se concentração de cipós com $\mathrm{DAP} \geq 10$ $\mathrm{cm}$ representando $1 \%$ do total de indivíduos registrados.

\section{As plantas com DAP $\geq 10 \mathrm{~cm}$}

\section{1) Composição florística}

Foram registrados indivíduos (abundância) por hectare, incluindo cipós, lianas e palmeiras, estando este resultado na média dos obtidos para a região amazônica, conforme Rankin-de-Mérona et al. (1992: 618 individuos), Valencia et al. (1994: 693), Tello (1995: 747), Amaral (1996: 747). Estes indivíduos estão distribuídos em 47 famílias, 118 gêneros e 145 espécies (Tab. 1). Com base nestes resultados, verificou-se que a área possui alta representatividade, quanto ao número de familias e gêneros, porém o número de espécies é baixo para o Estado do Amazonas. Dos 741 indivíduos, 629 $(83,8 \%)$ estão concentrados em 15 familias; os outros 112 distribuem-se entre as 32 familias restantes, demonstrando alta densidade em algumas familias.

Dentre as 15 familias mais abundantes, 5 detêm $50 \%$ dos individuos, sendo estas: Lecythidaceae, com 101 individuos; Burseraceae, 89; Chrysobalanaceae, 75; Caesalpiniaceae, 56; e Sapotaceae, 50. Analisando a densidade das familias, verifica-se que a familia Lecythidaceae é a que contém maior número de indivíduos, representando $13,6 \%$ do total. A alta densidade desta familia é também mencionada por outros estudiosos da Amazônia (Rodrigues, 1967; Prance et al., 1976; Campbell et al., 1986; Absy et al., 1986/87; Mori \& Boom, 1987; Salomão \& Lisboa, 1988; Rankin-deMérona et al., 1992). Esta família, segundo Mori \& Prance (1990), abrange desde pequenas a grandes árvores, crescendo em matas higrófilas (vegetação com alto grau de umidade), em altitude abaixo de $1000 \mathrm{~m}$, condições estas encontradas no rio Uatumã.

Analisando o número de indivíduos por espécie (abundância), verificou-se que 20 espécies detêm 423 individuos (Tab. 2), representando mais de $50 \%$ da densidade total, enquanto as 125 espécies restantes apresentam 318 individuos. Dentre as espécies mais abundantes, Protium apiculatum (Burseraceae) apresenta 84 individuos, sendo esta responsável por $11,3 \%$ da densidade total. Existe alta densidade em poucas espécies, haja vista que 82 espécies encontram-se representadas apenas por um ou dois indivíduos.

\section{2) Riqueza de espécies}

Das 145 espécies registradas, 13 famílias detiveram 87 espécies, correspondendo a $59,3 \%$ da riqueza de 
Tabela 1. Relação das espécies com DAP $\geq 10 \mathrm{~cm}$, nome popular, hábito e sua aplicabilidade econômica e/ou ecológica na floresta no rio Uatumã, AM.

\begin{tabular}{|c|c|c|c|c|}
\hline Família & Espécies & Nome popular & Hábito & Aplicaçăa \\
\hline \multirow[t]{3}{*}{ Anacardiaceae } & Anacardium giganteum Hancock ex Engl. & cajuí & árvore & ecológico \\
\hline & Anacardium parvifolium Ducke & cajuí & " & madeireiro \\
\hline & Tapirira guianensis Aubl. & pau-pombo & " & ecológico \\
\hline \multirow[t]{5}{*}{ Annonaceae } & Bocageopsis multiflora (Mart) R.E. Fries & & árvore & " \\
\hline & Duguettia sp. & & * & " \\
\hline & Guatteria discolor R. E. Fries & envira-fofa & " & " \\
\hline & Guatteria procera R. E. Fries & envira-amarela & “ & 4 \\
\hline & Xylopia brasiliensis Spreng. & envira-vassourinha & * & " \\
\hline \multirow[t]{4}{*}{ Apocynaceae } & Aspidosperma album (Vahl) R.Ben & piquiá-marfim & árvore & madeireiro \\
\hline & Geissospermum sericeum (Sagot) Benth & acariquara-branca & “ & medicinal \\
\hline & Himatanthus sucuuba (Spr.) Wood & sucúba & arvoreta & ecológico \\
\hline & Tabernaemontana maxima Markgraf & & & " \\
\hline \multirow[t]{2}{*}{ Arecaceae } & Maximiliana maripa (Aubl.) Drude & inajá & palmeira & ecológico \\
\hline & Oenocarpus bacaba Mart. & bacaba & " & $\begin{array}{l}\text { ecológico / } \\
\text { econômico }\end{array}$ \\
\hline \multirow[t]{2}{*}{ Bignoniaceae } & Jacaranda copaia (Aubl.) D. G. Don & caroba & árvore & madeireiro \\
\hline & Tabebuia serratifolia (G. Don) Nichols. & pau-d'arco & " & " \\
\hline \multirow[t]{2}{*}{ Bambacaceae } & Pachira insignis (Sw) Sw. & & $"$ & $"$ \\
\hline & Scleronema micranthum Ducke & cardeiro & $"$ & " \\
\hline Boraginaceae & Cordia goeldiana Hub. & frejió & ârvore & $\begin{array}{l}\text { ecológico/ } \\
\text { econômico }\end{array}$ \\
\hline \multirow[t]{2}{*}{ Burseraceae } & Hemicrepidospermum rhoifolium (Benth.) Sw. & breu-branco & árvore & ecológico \\
\hline & Protium apiculatum Swartz & breu-vermelho & “ & econômico \\
\hline \multirow[t]{10}{*}{ Caesalpiniaceae } & Bauhinia macrostachya Benth. & cipó & cipó & ecológico \\
\hline & Bauhinia splendens H.B.K. & cipó & “ & * \\
\hline & Campsiandra comosa (Benth.) Cowan & acapurana & 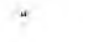 & madeireiro \\
\hline & Cassia lucens Vog. & & $"$ & ecológico \\
\hline & Copaifera multijuga Hayne & copaíba & árvore & medicinal \\
\hline & Cynometra spruceana Benth, & & 4 & \\
\hline & Dialium guianense (Aubl.) Sandw. & jutai-pororoca & " & ecológico \\
\hline & Macrolobium multijugum Benth. & arapari & * & madeireiro \\
\hline & Peltogyne paniculata Benth. & mulateiro & “ & " \\
\hline & Sclerolobium melanocarpum Ducke & taxi-vermelho & " & ecológico \\
\hline
\end{tabular}


Tabela 1 - Continuação

\begin{tabular}{|c|c|c|c|c|}
\hline & Tachigali myrmecophila (Ducke) Ducke & taxi-preto & " & * \\
\hline & Tachigali paniculata Aubl. & $\operatorname{taxi}$ & " & madeireiro \\
\hline Caryocaraceae & Caryocar glabrum (Aubl.) Pers. & piquiarana & " & " \\
\hline & Caryocar villosum (Aubl.) Pers. & piquiá-verdadeiro & “ & * \\
\hline Cecropiaceae & Cecropia sp. & embaúba-benguê & " & ecológico \\
\hline & Pourouma longipendula Ducke & embaúba-torem & " & " \\
\hline Celastraceae & Goupia glabra Aubl. & cupiúba & $=$ & madeireiro \\
\hline & Maytenus guianensis Klotzch. & chichuasca & - & medicinal \\
\hline Chrysobalanaceae & Couepia racemosa Benth, ex Hook. & pajurá-de-cotia & arvoreta & ecológico \\
\hline & Hirtella racemosa Lam. & & árvore & " \\
\hline & Licania egleri Prance & & " & “ \\
\hline & Licania lalifolia Benth. ex Hook & macucu-sangue & " & “ \\
\hline & Parinari montana Aubl. & pajurá-da-mata & " & “ \\
\hline Clusiaceae & Calophyllum brasiliense Camb. & jacareúba & $"$ & madeireiro \\
\hline & Moronobea coccinea Aubl, & bacuri-bravo & " & econômico \\
\hline & Vismia guianensis (Aubl.) Choisy & lacre & " & $\begin{array}{l}\text { ecológico / } \\
\text { econômico }\end{array}$ \\
\hline Combretaceae & Buchenavia parvifolia Ducke & tanimbuca & “ & “ \\
\hline & Combretum aubletii DC. & & ejpó & ecológico \\
\hline Dilleniaceae & Doliocarpus brevipedicellatus Garcke & cipo & cipó & $\begin{array}{l}\text { ecológico/ } \\
\text { medicinal }\end{array}$ \\
\hline Elaeocarpaceae & Sloanea floribunda Spr. ex Benth. & urucurana & árvore & ecológico \\
\hline Euphorbiaceae & Conceveiba guianensis Aubl. & & árvore & ecológico \\
\hline & Croton lanjouwensis Jablonski & dima & * & " \\
\hline & Mabea caudata P. et $\mathrm{H}$. & & arvoreta & " \\
\hline & Pogonophora schomburgkiana Miers & & árvore & madeireiro \\
\hline & Senefeldera macrophylla Ducke & & $*$ & ecológico \\
\hline Fabaceae & Andira retusa (Lam.) H.B.K. & & árvore & madeireiro \\
\hline & Bocoa viridiflora (Ducke) Cowan & & & " \\
\hline & Bowdichia nitida Spr. ex Benth. & sucuplra- da-terra-firme & árvore & madeireiro \\
\hline & Derris floribunda (Benth.) Ducke & cipó & cipó & ecológico \\
\hline & Diplotropis purpurea Rich & & árvore & madeireiro \\
\hline & Dipteryx odorata (Aubl.) Willd. & cumaru & $"$ & “ \\
\hline & Hymenolobium modestum Ducke & & a & " \\
\hline & Machaerium leiophyllum (DC.) Benth. & cipó & сірó & ecológico \\
\hline & Platymiscium duckei Huber & macacaúba & ârvore & madeireiro \\
\hline & Pterocarpus sp. & mututi & * & " \\
\hline & Swartzia corrugata Benth. & coraçāo-de-negro & $"$ & “ \\
\hline Hippocrateaceae & Salacia polyanthomaniaca Barb. Rodr. & сірó & cipó & ecológico \\
\hline
\end{tabular}


Tabela 1 - Continuação

\begin{tabular}{|c|c|c|c|c|}
\hline \multirow[t]{2}{*}{ Humiriaceae } & Duckesia verrucosa (Ducke) Cuatr. & uxi-coroa & árvore & madeireiro \\
\hline & Sacogiottis mattogrossensis Malme & & árvore & ecológico \\
\hline \multirow[t]{7}{*}{ Lauraceae } & Aniba panurene (Meissn.) Mez & louro-amarelo & árvore & madeireiro \\
\hline & Dicypelium manauense W. Rodr. & louro-preto & $n$ & " \\
\hline & Licaria aritu Ducke & louro & " & * \\
\hline & Mezilaurus itauba (Meissn.) Taub, ex Mez & itaúba & " & “ \\
\hline & Sextonia rubra van der Werff. & louro-gamela & $\cdot$ & * \\
\hline & Ocotea myriantha Mez & louro-abacate & $"$ & * \\
\hline & Ocotea canaliculata Mez & & " & $"$ \\
\hline \multirow[t]{5}{*}{ Lecythidaceae } & Bertholletia exce/sa Humb. \& Bonpl. & castanha-do-brasil & " & $\begin{array}{l}\text { madeireiro } \\
\text { econômico }\end{array}$ \\
\hline & Eschweilera coriacea (A.P.DC.) Mori & ripeiro-preto & $=$ & ecológico \\
\hline & Eschweilera albiffora (A.DC,) Miers & & 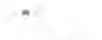 & $=$ \\
\hline & Lecythis jarana A.C. Smith & & àrvore & ecológico \\
\hline & Lecythis latifolium (A.C.Sm.) Knuth & jarana & $"$ & " \\
\hline Linaceae & Roucheria punctata Ducke & azeitona-da-mata & árvore & $"$ \\
\hline Malpighiaceae & Byrsonima lancifolia Juss. & murici & árvore & " \\
\hline \multirow[t]{2}{*}{ Melastomataceae } & Miconia elaeagnoides Cogn. & orelha-de-burro & árvore & ecológico \\
\hline & Miconia regelii Cong. & & $"$ & $"$ \\
\hline \multirow[t]{2}{*}{ Memecylaceae } & Mouriri duckeana Morley & & árvore & ecológica \\
\hline & Mouriri plasschaerti Pulle & muiraúba & $"$ & $"$ \\
\hline \multirow[t]{3}{*}{ Meliaceae } & Guarea carinata Ducke & jitó & árvore & madeireiro \\
\hline & Trichilia micrantha Benth. & & " & ecológico \\
\hline & Trichilia paniculata Aubl. & & " & $"$ \\
\hline \multirow[t]{3}{*}{ Menispermaceae } & Abuta grandifolia (Mart.) Sandw. & & cipó & ecológico \\
\hline & Abuta panurensis Eichl. & & cipó & $\cdot$ \\
\hline & Abuta sp. & & cipó & $"$ \\
\hline \multirow[t]{10}{*}{ Mimosaceae } & Enterolobium schomburgkil Benth. & orelha-de-macaco & árvore & ecológico \\
\hline & Inga cayennensis Sagot. ex Benth. & ingá-vermelho & $\alpha$ & $"$ \\
\hline & Inga sp. & ingá-branca & * & - \\
\hline & Zygia racemosum (Ducke) Barneby \& Grimes & angelim-rajado & " & madeireiro \\
\hline & Mimosa spruceana Benth. & cipó & cipó & ecológico \\
\hline & Parkia multijuga Benth. & & árvore & madeireiro \\
\hline & Parkia pendula Benth. ex Wall & visgueiro & * & ecológico \\
\hline & Piptadenia suaveolens Miq. & & • & 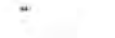 \\
\hline & Pithecellobium dinizii Ducke & & $"$ & madeireiro \\
\hline & Pithecellobium sp. & & * & ecológico \\
\hline Monimiaceae & Siparuna amazonica Mart. & capitiú & árvoreta & ecológico \\
\hline Moraceae & Brosimum potabile Ducke & amapá & ánore & madeireiro \\
\hline
\end{tabular}


Tabela 1 - Continuação

\begin{tabular}{|c|c|c|c|c|}
\hline & Brosimum rubescens Taub. & pau-rainha & $"$ & " \\
\hline & Clarisia racemosa $\mathrm{B}$. et $\mathrm{P}$. & guariúba & árvore & madeireiro \\
\hline & Helicostylis podogyne Ducke & & " & " \\
\hline & Pseudolmedia laevis (R.\& P.) Macbr. & pama & “ & “ \\
\hline \multirow[t]{4}{*}{ Myristicaceae } & Iryanthera tricomis Ducke & & ávore & ecológico \\
\hline & Virola elongata (Benth.) Warb. & ucuúba & " & madeireiro \\
\hline & Virota multicostata Ducke & ucuúba-vermelha & " & " \\
\hline & Virola multinervia Ducke & ucuúba-preta & " & " \\
\hline \multirow[t]{8}{*}{ Myrtaceae } & Calyptranthes bipennis Berg & ubaia & " & ecológico \\
\hline & Calyptranthes macrophylla Berg & & " & " \\
\hline & Eugenia patrisii Vahl & ubará & arvoreta & " \\
\hline & Eugenia cucculata Amsh. & & " & $"$ \\
\hline & Eugenia cuspidifolia DC. & mirtácea-amarela & arvoreta & ecológico \\
\hline & Eugenia pseudopsidium Jacq. & & " & “ \\
\hline & Eugenia sp.(1) & & $"$ & " \\
\hline & Eugenia sp.(2) & & " & " \\
\hline Nyctaginaceae & Neea sp. & joăo-mole & árvore & ecológico \\
\hline Olacaceae & Minquartia guianensis Aubl. & acariquara-roxa & árvore & madeireiro \\
\hline Polygalaceae & Securidaca bialata Benth. & cipó & cipó & ecológico \\
\hline \multirow[t]{2}{*}{ Polygonaceae } & Coccolobasp. & & arvoreta & ecológico \\
\hline & Indet. & & * & " \\
\hline \multirow[t]{2}{*}{ Rubiaceae } & Duroia macrophylla Huber & & arvoreta & ecológico \\
\hline & Randia sp. & cipó & cipó & “ \\
\hline \multirow[t]{2}{*}{ Sapindaceae } & Talisia cupularis Radlk. & & arvoreta & ecológico \\
\hline & Talisia guianensis Aubl. & & $"$ & “ \\
\hline \multirow[t]{5}{*}{ Sapotaceae } & Chrysophyllum prieurii A.DC. & abiurana-vermelha & árvore & ecológico \\
\hline & Micropholis mensalis (Baehni) Aubr. & 1 & * & $"$ \\
\hline & Micropholis multijuga Benth. & & " & " \\
\hline & Micropholis venulosa (Mart ex. Eichl.) Pier. & abiurana-branca & " & " \\
\hline & Pouteria guianensis Aubl. & & " & " \\
\hline Simaroubaceae & Simarouba amara Aubl. & marupá & árvore & madeireiro \\
\hline \multirow[t]{2}{*}{ Sterculiaceae } & Sterculia pruriens (Aubl.) K. Schum & chichá & árvore & ecológico \\
\hline & Theobroma speciosum Willd. & cacauí & " & " \\
\hline \multirow[t]{2}{*}{ Tiliaceae } & Apeiba echinata Gaert. & & " & " \\
\hline & Mollia sp. & & " & “ \\
\hline Verbenaceae & Vitex cymosa Bert. ex Spreng. & tarumã & $"$ & " \\
\hline \multirow[t]{2}{*}{ Violaceae } & Paypyarola sp. & & arvoreta & $"$ \\
\hline & Rinorea racemosa (Mart. et Zucc.) Ktz. & & " & * \\
\hline Vochysiaceae & Ruizterania albiflora Marcano-Berti & & árvore & * \\
\hline
\end{tabular}


Tabela 2. Relação das 20 espécies (DAP $\geq 10 \mathrm{~cm}$ ) com maior número de indivíduos e sua proporção em relação ao total na floresta do rio Uatumã, AM.

Nome Científico № de individuos Proporção em relação ao total $(\%)$

Protium apiculatum

84

11,33

Eschweilera coriacea

57 7,69

Licania latifolia

34 4,59

Licania egleri 32 4,31

Chrysophyllum prieurii 28 3,77

Eschweilera albiflora 25

Xylopia brasiliensis

Helicostylis podogyne

Guatteria discolor

Virola multicostata

Dicypelium manauense

Tachigali paniculata

Cynometra spruceana

Conceveiba guianensis

Eugenia cuspidifolia

Inga sp.

Micropholis venulosa

Scleronema micranthum

Trichilia micrantha

Sacoglottis mattogrossenis

Sub-total (20 espécies)

Outras 125 espécies

espécies em um hectare; as demais (59) encontram-se distribuidas entre as 34 famílias restantes, demonstrando que há famílias com alta concentração de espécies. Dentre estas destacam-se Caesalpiniaceae (12), Fabaceae (11),
Mimosaceae (10), Myrtaceae (8), Lauraceae (7), representando $33 \%$ da riqueza. Excetuando Myrtaceae, as demais estão de acordo com outros resultados apresentados para a Amazônia em geral. 
A curva espécie/área, que representa a expressão analítica da relação entre o incremento da área amostrada em uma comunidade e o número das espécies acumuladas (Figura 2), demonstra que o local não é muito diversificado, haja vista que essa curva tende a atingir a assíntota nos $10.000 \mathrm{~m}^{2}$, indicando que o número de amostras foi suficiente para estimar a riqueza local. Tal fato não é muito comum para a floresta de terra firme, pois a tendência é de haver acréscimo de novas espécies nas últimas parcelas (Salomão \& Lisboa, 1988; Tello, 1995; Amaral, 1996). Uma explicação provável é a não ocorrência de variações nas comunidades florísticas na área estudada.

$\mathrm{Na}$ análise comparativa entre as famílias com maior riqueza de espécies e densidade, verificou-se que apenas Caesalpiniaceae, com 12 espécies e 56 indivíduos, mantêm uma relação direta entre densidade e riqueza de espécies, enquanto que Lecythidaceae, com a maior densidade (101 individuos), não mantêm esta premissa, apresentando apenas 5 espécies. Este resultado evidencia que nem sempre a familia com maior densidade é a que detêm maior riqueza de espécies. Entretanto, em outros estudos realizados na Amazônia (Campbell et al., 1986; Mori et al., 1989; Prance, 1990; Ribeiro et al., 1994; Tello, 1995; Amaral, 1996), observase que pode haver relação entre riqueza de espécies e densidade nas familias.

\section{3) Parâmetros estruturais}

a) Índice de Valor de Importância

O Índice de Valor de Importância das espécies (IVI) é usado para quantificar a participação e distribuição espacial das mesmas, dentro de determinada comunidade (Lamprecht, 1964). Na área estudada, Protium apiculatum (IVI $=25,5$ ) representou $9 \%$ do IVI total e Eschweilera coriacea $($ IVI $=17,6)$ representou $6 \%$. Estas duas espécies foram as mais importantes, indicando que a área é ecologicamente dominada pelas mesmas. De fato, elas encontramse em primeiro e segundo lugar, respectivamente, em todos os índices relativos das espécies, indicando igualdade de distribuição (freqüência relativa), dominância de cobertura na superfície (dominância relativa) e predominância de indivíduos (abundância relativa) (Tab. 3).

Fazendo uma análise comparativa entre as 20 espécies com maior número de indivíduos e as com maiores IVI (Tabela 1 e Tabela 2), verificou-se que há uma sobreposição de 15 espécies, representando $75 \%$, demonstrando haver correlação entre abundância de individuos por espécie e o índice de valor de importância no local. Entretanto, a espécie Bertholletia excelsa, que está representada por um indivíduo, encontra-se na décima segunda posição no IVI, devido à área basal de $5,2 \mathrm{~m}^{2}$, enquanto que as espécies Eugenia cuspidifolia, Micropholis venulosa, Trichilia micrantha e Sacoglottis mattogrossensis, que detêm entre 9 e 8 indivíduos respectivamente, não constam entre as 20 espécies com maior IVI devido estas possuírem baixa freqüência e dominância. 


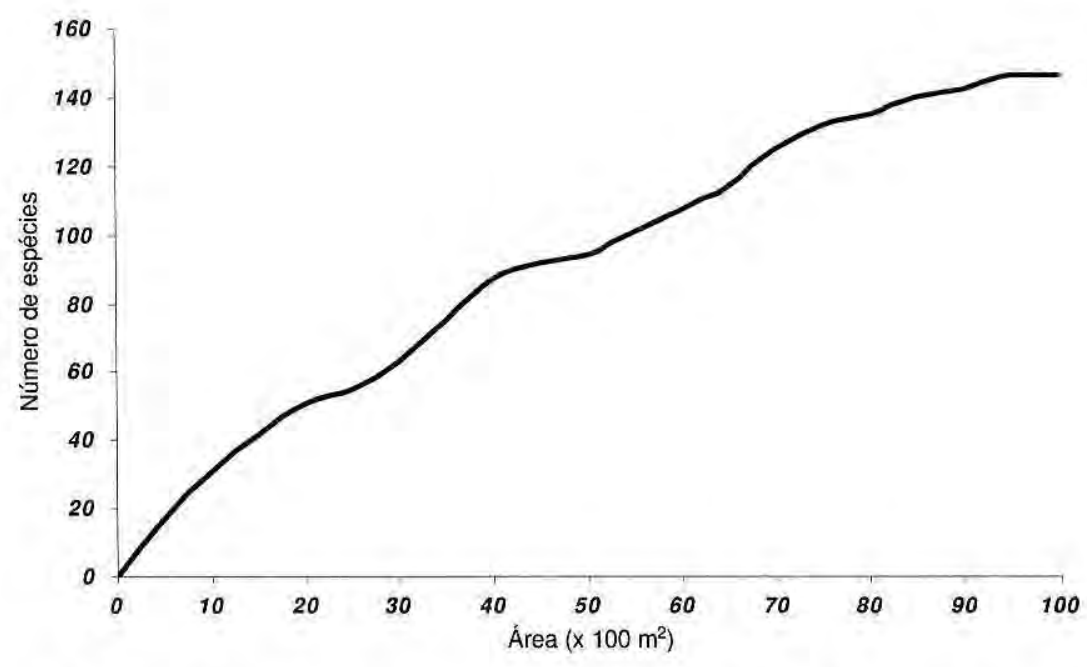

Figura 2. Curva cumulativa das espécies com DAP $\geq 10 \mathrm{~cm}$ na área estudada no rio Uatumã, AM.

\section{b) Classes de Diâmetro}

Analisando a distribuição dos indivíduos por classe de diâmetro, verificou-se que nas primeiras classes (10-20 e 20-30 cm) há concentração de 642 indivíduos, representando $86,6 \%$ do total de indivíduos registrados, comprovando que esta floresta é composta na sua maioria de indivíduos finos, sendo, portanto, imprópria para exploração de madeira (Figura 3). Por outro lado, apesar desta floresta ser composta por indivíduos finos, não significa que esta seja uma floresta jovem, pois quase todas as classes diamétricas estão representadas, excetuando a classe de $75-80 \mathrm{~cm}$ e, de acordo com Finol (1976), uma distribuição diamétrica decrescente, com a maioria de classes representadas, indica uma floresta madura, estabilizada e com a perpetuação das espécies garantida.
As espécies Bertholletia excelsa, Scleronema micranthum, Chrysophyllum prieurii e Diplotropis purpurea foram consideradas de grande porte, por terem ocorrido somente nas classes de DAP maiores a $55 \mathrm{~cm}$. Por outro lado, espécies como Xylopia brasiliensis, Mabea caudata, Randia sp, Talisia cupularis e Hirtella racemosa foram consideradas exclusivas do estrato médio, devido ocorrerem nas classes de DAP inferior a $25 \mathrm{~cm}$.

\section{$O$ estrato inferior}

No estrato inferior (sub-bosque) foram catalogados 599 indivíduos, distribuídos em 33 famílias, 59 gêneros e 59 espécies. A classe de altura dominante em termos quantitativos é a de 0-0,50 m, com 400 indivíduos. Esta categoria é considerada como plântulas (pequenas 
Tabela 3. Relação das 20 espécies com DAP $\geq 10 \mathrm{~cm}$ ordenados pelo IVI, junto com os parâmetros de frequeência, dominância e abundância (relativa), na floresta do rio Uatumã, AM.

\begin{tabular}{lcccc}
\hline Nome Cientfíico & FRrel & DOMrel & ABrel & I.V.I. \\
\hline Protium apiculatum & 3,58 & 7,46 & 11,34 & 22,37 \\
Eschweilera coriacea & 3,58 & 6,29 & 7,69 & 17,56 \\
Licania latifolia & 3,10 & 4,08 & 4,59 & 11,77 \\
Licania egleri & 3,10 & 3,58 & 4,32 & 11,00 \\
Chrysophyllum prieurii & 2,39 & 3,79 & 3,78 & 9,96 \\
Eschweilera albiflora & 1,91 & 3,42 & 3,37 & 8,70 \\
Xylopia brasiliensis & 2,39 & 1,58 & 2,43 & 6,39 \\
Sclerolobium melanocarpum & 0,72 & 4,49 & 1,08 & 6,28 \\
Helicostylis podogyne & 2,63 & 1,39 & 2,16 & 6,18 \\
Virola multicostata & 2,86 & 1,11 & 1,89 & 5,86 \\
Dicypelium manauense & 1,43 & 2,73 & 1,62 & 5,78 \\
Bertholletia excelsa & 0,24 & 5,24 & 0,13 & 5,62 \\
Cynometra spruceana & 0,95 & 2,88 & 1,48 & 5,32 \\
Guatteria discolor & 1,43 & 1,32 & 1,89 & 4,65 \\
Couepia racemosa & 1,43 & 2,04 & 1,08 & 4,55 \\
Dialium guianense & 0,95 & 2,50 & 0,81 & 4,26 \\
Conceveiba guianensis & 1,67 & 1,34 & 1,22 & 4,22 \\
Scleronema micranthum & 1,19 & 1,81 & 1,22 & 4,21 \\
Tachigali paniculata & 1,19 & 1,21 & 1,62 & 4,03 \\
Mabea caudata & 0,72 & 2,26 & 0,94 & 3,92 \\
Sub-total (20 espécies) & 37,47 & 60,64 & 53,04 & 151,14 \\
Outras 125 espécies & 62,53 & 39,36 & 46,96 & 148,86 \\
Total & 100,00 & 100,00 & 100,00 & 300,00 \\
\hline & & & &
\end{tabular}

plantas recém-nascidas), segundo a classificação das formas de vida vegetal proposta por Aubréville (1963). Nas classes de altura subseqüentes houve um decréscimo significativo quanto ao número de indivíduos, pois estas, somadas, representaram $33,2 \%$, contra os $66,8 \%$ da classe de menor tamanho (Fig. 4).

A forma de vida mais abundante em todas as categorias de tamanho foi a das árvores, representanto $38,7 \%$ do total. Entretanto, os cipós estão presentes em toda a área estudada e, 


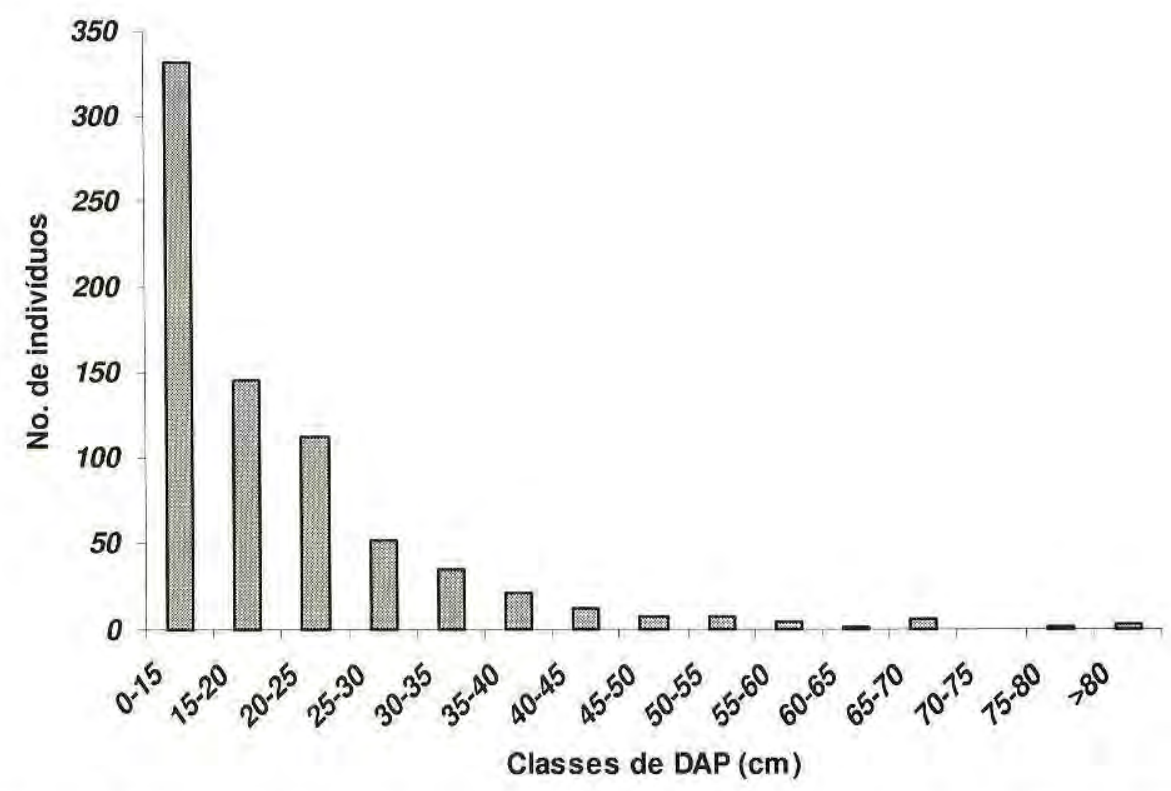

Figura 3. Distribuição dos indivíduos nas classes de DAP na área estudada no rio Uatumã, AM.

por ser esta uma forma de vida bastante agressiva, especialmente após a interferência antrópica, eles tendem a dominar no primeiro estágio da sucessão da floresta. Desta forma de vida, as espécies mais abundantes foram Bauhinia macrostachya (69), Arrabidaea sp. (33) e Derris floribunda (13). Outro fato observado foi quanto ao número de individuos (plântulas) de árvores na primeira classe de altura (170 plântulas) e a relação com as que se tornaram plantas estabelecidas ( $3 \mathrm{~m}$ de altura), entre as quais foram constatadas apenas 22 indivíduos, enquanto que $87 \%$ pereceram. Resultados semelhantes foram encontrados por Higuchi et al. (1985) na Amazônia Central.

As espécies que apresentaram maior número de indivíduos foram
Protium apiculatum (73), Cynometra spruceana (36), Tachigali paniculata (34) e Sloanea floribunda (29), sendo estas de dossel. Outras espécies que apresentaram um número expressivo de indivíduos foram Pariana sp. (78), Ischnosiphon ovatus (45), Eugenia sp. (27) e Psychotria sp. (22), representando $28,7 \%$ dos indivíduos e $25,4 \%$ das espécies registradas neste estrato. Estas espécies completam seu ciclo de vida no estrato inferior; por conseguinte, são típicas de sub-bosque e não toleram incidência solar direta, sendo as primeiras a serem afetadas, drasticamente com a interferência antrópica, como por exemplo a extração madeireira.

\section{CONCLUSÕES}

O local estudado, apesar de apresentar um número razoável de 
espécies por hectare (145), não pode ser considerado rico em espécies vegetais quando comparado com outros locais da Amazônia Central. Entretanto, ao analisar as espécies po: meio do Índice de Valor de

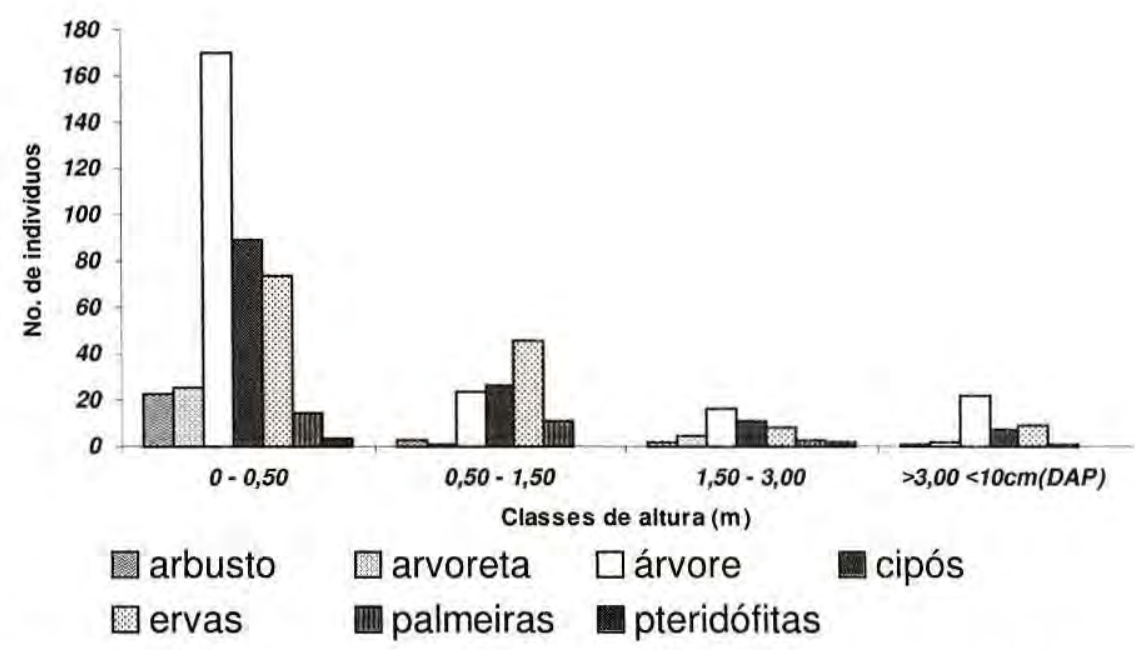

Figura 4. Distribuição dos indivíduos, por classe de altura, das diferentes formas de vida (hábito) do estrato inferior na área estudada no rio Uatumã, AM.

Importância (IVI), verificou-se que algumas dessas mantêm afinidades com outros resultados da Amazônia Central, como é o caso das espécies Protium apiculatum e Eschweilera coreacea, que estão entre as 20 espécies com maior valor de IVI. A análise comparativa dos parâmetros densidade ( $\mathrm{n}^{\circ}$ ind./ha) e riqueza de espécies ( $\left.\mathrm{n}^{\circ} \mathrm{spp} . / \mathrm{ha}\right)$ ao nível familiar, mostrou que nem sempre há uma relação direta entre estes parâmetros, pois verificou-se que a familia com maior densidade não é a que apresenta maior riqueza de espécies. Os estudos do estrato inferior revelaram que algumas espécies consideradas por profissionais de manejo florestal como espécies que irão perpetuar a floresta não sobreviverão a ação antrópica por se tratar de espécies próprias de subbosque e, em geral, não toleram a incidência direta de luz.

\section{Bibliografia citada}

Absy, M.L.; Prance, G.T.; Barbosa, E.M. 1986/ 87. Inventário florístico de floresta natural na área da estrada Cuiabá-Porto Velk (BR-364). Acta Amazonica, 1/ 17(único):85-121.

Albuquerque, O.R. de 1922. Reconhecimentus geológicos no valle do Amazonas. Boletir: n² 3. Serviços geológicos e mineralógico; do Brasil, Ministério da Agricultura, Ind. e Comércio. Rio de Janeiro. 84p.

Amaral, I.L. 1996. Diversidade floristica em floresta de terra firme, na região do rio Urucu, AM. Dissertação de Mestrado, INPA/FUA, Manaus, AM. 104p.

Aubréville, A. 1963. Classification des formes biologiques des plantes vasculaires in milieu tropicale. Adansonia, 3(2):221-226. 
Campbell, D.G.; Daly, D.C.; Prance, G.T.; Maciel, U.N. 1986. Quantitative ecological inventory of terra firme and varzea tropical forest on the rio Xingu, Brazilian, Amazon. Brittonia, 38(4):369-393.

Condit, R.; Hubell, S.P.; Lafrankie, J.V..1996. Species-area and species-individual relationships for tropical trees: A comparision of three 50-ha plots. J. Ecology, 84(4):549-562.

Finol, U.H. 1976. Estúdio fitossociológico de las unidades 2 e 3 de la Reserva Florestal de Caparo, estado de Barida. Acta Botanica Venezuelana, 10(14):15-103.

Higuchi, N.; Jardim, F.C.S.; Santos, J.; Alencar, J.C. 1985. Inventário diagnóstico da regeneração natural. Acta Amazonica, 15(1-2):199-233.

Lamprecht, T.H. 1964. Ensayo sobre la estructura floristica del parte sur-oriental del bosque universitário "El Caimital"Estado Baridas, Rev. For. Venez., 7(1011):77-119.

Magurran, A.E. 1988. Ecological diversity and its measurement. Cambrigde University Press, Cambridge. 179p.

Mori, S.A.; Boom, B.M. 1987. The forest. In: Prance G.T.; Mori, S.A. (Eds.). The Lecythidaceae of a lowland Neotropical forest: La Famée Mountain, French Guiana. Memoirs of the New York Botanical Garden, $\mathrm{n}^{\circ} 44$, The New York Botanical Garden, Bronx, NY. p. 9-29

Mori, S.A.; Rabelo, B.V.; Tsou, C.H.; Daly, D.C. 1989. Composition and structure of an eastern amazonian forest at Camaipi, Amapa, Brazil. Bol. Mus. Para. Emilio Goeldi, sér. Bot., 5(1):3-29.

Mori, S.A.; Prance, G.T. 1990. Lecythidaceae - part II. Flora Neotropica, Monograph 21(II). The New York Botanical Garden, Bronx, NY. 375p.

Prance, G.T.; Rodrigues, W.A.; Silva, M.F. da 1976. Inventário florestal de 1 ha de mata de terra firme, $\mathrm{Km} 30$ da Estrada ManausItacoatiara. Acta Amazonica, 6(1):9-35.

Prance, G.T. 1990. The floristic composition of the forests of Central Amazonian Brazil.
In: Gentry, A. (Ed.). Four Neotropical forests. Yale University Press, New Haven, CT. p.112-140.

Rankin-de-Mérona, J.; Prance, G.T.; Hutchings, R.W.; Silva, M.F. da; Rodrigues, W.A.; Venling, M.E. 1992. Preliminary results of a large-scale tree inventory of upland rain forest in the Central Amazon. Acta Amazonica, 22(4):485-492.

Ribeiro, J.E.L.S.; Nelson, B.W.; Silva, M.F. da; Martins, L.S.S,; Hopkins, M. 1994. Reserva Florestal Ducke: Diversidade e composição da flora vascular. Acta Amazonica, 24(1/2):19-30.

Rodrigues, W.A. 1967. Inventário florestal piloto ao longo da estrada ManausItacoatiara, estado do Amazonas: dados preliminar. In: Lent Herman (Ed.). Atas do Simpósio sobre a Biota Amazônica. Vol. 7. Conselho Nacional de Desenvolvimento Científico e Tecnológico (CNPq), Rio de Janeiro, Guanabara. p.257-267.

Salomão, R.P.; Lisboa, P.L. 1988. Análise ecológica da vegetação de uma floresta pluvial tropical de terra firme, Rondônia. Bol. Mus. Para. Emilio Goeldi, sér. Botânica, 4(4):195-234.

Silva, M.F.; Lisbôa, P.L.B.; Lisbôa, R.C.L. 1977. Nomes vulgares de plantas amazônicas. Instituto Nacional de Pesquisas da Amazônia (INPA), Conselho Nacional de Desenvolvimento Científico e Técnológico (CNPq), Belém, Pará. 222p.

Tello, J.C.R. 1995. Aspectos fitossociológicos das comunidades vegetais de uma toposseqüencia da Reserva Florestal Ducke do INPA. Tese de Doutorado, INPA/FUA, Manaus, AM. 335p,

Valencia, R.; Balslev, H.; Miño, C.G.P.Y. 1994. High tree alpha diversity in Amazonian Ecuador. Biodiversity and Conservation, $3: 21-28 \mathrm{p}$

Veloso, H.P.; Rangel Filho, A.L.R.; Lima, J.C.A. 1991. Classificação da vegetação brasileira, adaptada a um Sistema Universal. Fundação Instituto Brasileiro de Geografia e Estatística - IBGE, Rio de Janeiro, Brasil. 124p. 